\title{
No Sudden Audio Switch -- Preventing discontinuous POI audio playing in LBS
}

\author{
Ruochen $\mathrm{Si}^{\text {a, }}{ }^{*}$, Masatoshi Arikawa ${ }^{\mathrm{b}}$, Hideki Kaji ${ }^{\text {a, }}$, Tianqi Xia ${ }^{\text {a, }}$, Shibasaki Ryosuke ${ }^{\text {a }}$ \\ ${ }^{a}$ Center for Spatial Information Science, The University of Tokyo, si@csis.u-tokyo.ac.jp, kaji@csis.u-tokyo.ac.jp, xiatianqi@csis.u- \\ tokyo.ac.jp, shiba@csis.u-tokyo.ac.jp \\ ${ }^{b}$ Graduate School of Engineering Science, Faculty of Engineering Science, Akita University, arikawa@ie.akita-u.ac.jp
}

Keywords: POI audio, composed audio, buffered geofence, LBS

\begin{abstract}
:
Many LBS applications provide automatic audio playing functions for introducing POI's. Appropriate automatic audio playing can improve users' expressions during traveling with LBS and in some degree prevent walking-smartphone. However, the problem of discontinuity of audio playing on the contrary affects users' expressions. One main reason that cause the discontinuity of audio playing is the sudden switch of audios, usually caused by a user entering the geofence of another POI while the audio of current POI is under playing. This paper proposed a preliminary approach to prevent the sudden switch of the audio instruction of POI's by making adjustments on the POI's audios and geofences.
\end{abstract}

Current main approach for automatic POI audio playing is using a geofence of the POI as a spatial trigger to control the audio playing. This simple approach may cause sudden switch of audio playing, which causes negative users' expressions. As shown in Figure 1, a user's moving trajectory frequently crosses geofences of two POI's, which will result in a frequent switch of POI's audios with neither completely played. The inflexible audio files and geofences are the main reasons causes the sharp audio switches and we are going to solve the problem by making the audio files and geofences flexible.

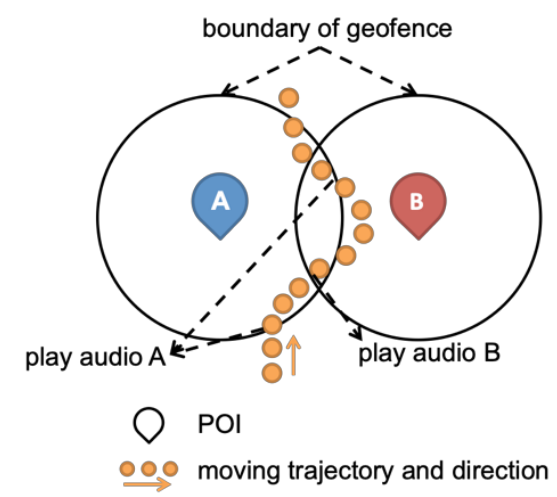

Figure 1. Problem of sudden audio switches when automatically play POI's audios.

Conventional POI audio is made of one integrated file. As shown in Figure 2, we separate the POI audio content into several audio files, with each file consists one sentence or one short paragraph, and we call it composed audio. The files of a composed audio will be played in sequence, and the basic rule of playing a composed audio is not stopping until one file is completely played, which ensures that the composed audio will stop naturally after a complete sentence or paragraph. We define four important events for automatic playing composed audios. Start playing means start to play a composed audio from the beginning. It usually happens when a user enters a geofence of a POI. Stop playing means to stop a composed audio when finishing playing the current audio file. It usually happens when a user enters the geofence of another POI. Prepare playing means to start playing a composed audio when finishing playing the composed audio that is under playing. It usually happens when enters the geofence of a second POI. Cancel playing means to cancel the prepare playing status of a composed audio. It happens when a composed audio of a POI is in prepare playing status but the user leaves the geofence of the current POI or enters the geofence of another POI before the composed audio starting playing. 


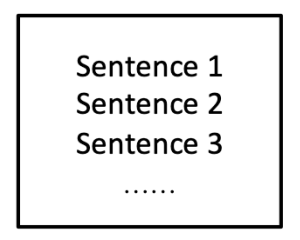

(a) Integrated audio file

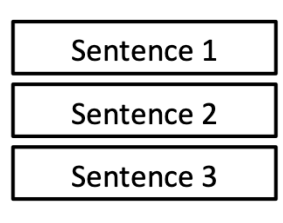

(b) Separated audio files

Figure 2. Structures of conventional integrated audio file and separated audio files for composed audio of a POI.

By introducing buffers to a geofence (Tokita, Arikawa, $\mathrm{Si}$ et al, 2018), it makes a geofence flexible, and we note the geofences with buffers as the buffered geofences. As shown in Figure 3, here we add an outer buffer to the geofence in Figure 1 to improve stability of POI's audio play. An object has two status related to the buffered geofence: out and in, and it has two events related to the buffered geofence: enter and leave. The enter event occurs when the object is out of the buffered geofence and crosses the geofence's boundary; the leave event occurs when the object is in the buffered geofence and crosses the geofence's outer buffer. The result is that even though the user is frequently crossing the boundaries of the buffered geofences, he/she only enters each buffered geofences once, thus the audios of each POI will play once and will not be frequently switched.

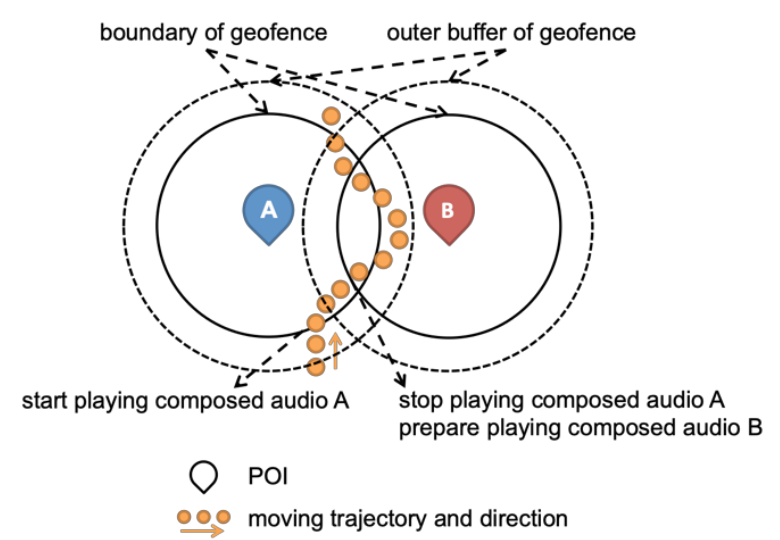

Given a POI set $\mathrm{S}=\left\{\mathrm{POI}_{1}, \mathrm{POI}_{2} \ldots \mathrm{POI}_{\mathrm{n}}\right\}$, as shown in Table 1, by combining the composed audio event and buffered geofence event, we are able to play POI's audios naturally without sudden or switches of the audios.

\begin{tabular}{|c|c|c|}
\hline & Enter buffered geofence of POI $_{\mathrm{i}}$ & Leave buffered geofence of POI $_{\mathrm{i}}$ \\
\hline POI $_{\mathrm{m}}(m=i)$ & Start/Prepare playing & Cancel playing \\
\hline POI $_{\mathrm{n}}(n \neq i)$ & Stop/Cancel playing & - \\
\hline
\end{tabular}

Table 1. combination of composed audio events and buffered geofence events.

In this paper we introduced an approach to prevent discontinuous POI's audio playing by composed audios and buffered geofences. This research is still preliminary, and it needs more researches on how to separate POI audios: too fragmented separation of POI audio may still make users feel an unnatural finish of an audio, and too generalized separation may result in more time costed before stopping a composed audio. Also, the current rules for playing composed audios based on the buffered geofences are relatively simple, which may cause unbalanced audio play. For example, when a user enters the buffered geofence of POI_A and soon enters the buffered geofence of POI_B, the time for playing composed audio of POI_A will be short. In the future, we plan to estimate the overall time for candidate audios to be played and balance the time for each of them. 